\title{
Accurate Palmprint Recognition Using Spatial Bags of Local Layered Descriptors
}

\author{
Yufei Han, Tieniu Tan, and Zhenan Sun \\ Center for Biometrics and Security Research, National Laboratory of Pattern Recognition \\ Institute of Automation, Chinese Academy of Sciences \\ \{yfhan, tnt, znsun\}@nlpr.ia.ac.cn
}

\begin{abstract}
State-of-the-art palmprint recognition algorithms achieve high accuracy based on component based texture analysis. However, they are still sensitive to local variations of appearances introduced by deformation of skin surfaces or local contrast variations. To tackle this problem, this paper presents a novel palmprint representation named Spatial Bags of Local Layered Descriptors (SBLLD). This technique works by partitioning the whole palmprint image into sub-regions and describing distributions of layered palmprint descriptors inside each sub-region. Through the procedure of partitioning and disordering, local statistical palmprint descriptions and spatial information of palmprint patterns are integrated to achieve accurate image description. Furthermore, to remove irrelevant and attributes from the proposed feature representation, we apply a simple but efficient ranking based feature selection procedure to construct compact and descriptive statistical palmprint representation, which improves classification ability of the proposed method in a further step. Our idea is verified through verification test on large-scale PolyU Palmprint Database Version 2.0. Extensive experimental results testify efficiency of our proposed palmprint representation.
\end{abstract}

\section{Introduction}

The past decade has witnessed a great progress of biometrics technology, which identifies different people by their physiological and behavioral difference, such as face, iris, retinal, gait and etc [1]. Palmprint recognition is a novel but promising member of biometrics. Large region of palm supplies amounts of discriminative line and texture patterns, which could be captured using a low resolution imaging device, such as a low-cost CCD camera [2]. Thanks to its high recognition accuracy and convenience for practical use, palmprint recognition provides a promising way for identity check. Palmprints normally refer to appearance properties of structure patterns of epidermis on palm regions, which are formed due to movement of fingers, structures of tissue and muscles gradually after birth and spread over different areas of palmprint images. Therefore, a key issue in palmprint recognition is to design an efficient image representation to depict the skin textures accurately. In previous work, component based texture analysis provides a reasonable framework for palmprint recognition [2-8]. Most state-of-the-art approaches [5-8] can be ranged into this framework. For one 
palmprint image, characteristics of palmprints inside each local patch are described using image filters, like multi-directional Gabor filter set [5][6] or orthogonal line ordinal filters [7]. Either phase [5] or magnitude [6][7] of filtering responses are used as local texture descriptors, which are rearranged into a feature matrix or template according to their spatial order. Feature matching is conducted by comparing texture features of corresponding patches pair-wisely in two palmprint images. Differences between local regions are accumulated to form a similarity measure between the two images. The component based framework makes good use of intrinsic properties of palmprints. As a result, this framework obtains good performance. However, it also suffers from variations of palmprint appearances between intra-class samples. The reason for such variation can be concluded into two aspects, as shown in Fig.1. For one thing, due to different postures of hands in practical conditions, captured palmprint images usually contain contraction or stretching of skin surfaces caused by movement of muscle tissues, which leads to deformation of skin textures. For another thing, variations of illumination settings over local region also change contrast information of palmprint appearances. For two palmprint images belonging to the same class, the variations of appearances easily lead to discrepancies between component based texture descriptions in corresponding regions, which consequently limits further increasing of descriptive power.

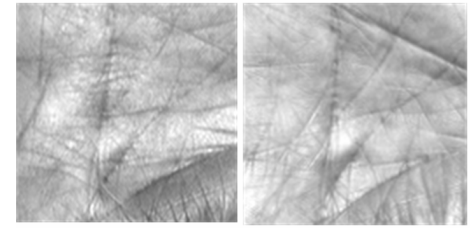

(a)

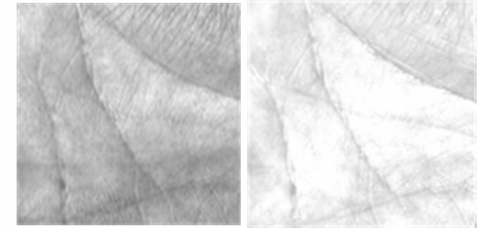

(b)

Fig. 1. Variations of palmprint appearances caused by (a) contraction or stretching of skin surfaces and (b) variations of illumination settings

Unlike component based methods, Bag-of-Feature model is a holistic statistical image representation [11][13]. General scheme of this approach is to represent an image by an orderless collection of local features, disregarding their spatial information. Bag-of-Feature model of one specific image is constructed by computing a histogram of votes, which counts occurrence frequency of local features in the image [11][13]. It makes an improvement in robustness of image description against local appearance deformations in nature. This model is popular in object recognition or scene categorization. Motivated by Bag-of-feature model, in this paper, we propose a statistical palmprint representation, called Spatial Bags of Local Layered Descriptors (SBLLD), as illustrated in Fig.2. We firstly construct local layered descriptors for palm lines and textures using multi-directional quadrature Gabor filters, which are both discriminative palmprint features. A tree-structured visual vocabulary of local layered descriptors is then generated and pruned without prior clustering on training data. Finally, the whole image is subdivided into non-overlapped sub-regions. Distributions of visual vocabulary within each sub-region are described following Bag-of-Feature framework and aggregated into a histogram sequence according to spatial order of sub-regions. 
Furthermore, we select the most discriminative attributes from histogram based palmprint features. With this helpful procedure, the proposed method achieves even higher accuracy with more compact feature subset. Our goal of this paper can be concluded into two aspects. For one thing, by compensating loss of spatial information with the subdividing scheme, we improve descriptive ability of statistical image model to be suitable for palmprint classification. For the other thing, by explicitly introducing the statistical image description into palmprint representation, more robust recognition against local variations of appearances are achieved compared with component based approaches.

Remainder of this paper is organized as follows: In Section.2, we give detailed description of the proposed method. Section.3 illustrates procedure of ranking based feature selection. Comprehensive experimental results are listed and analyzed in Section.4. Section. 5 concludes the whole paper.

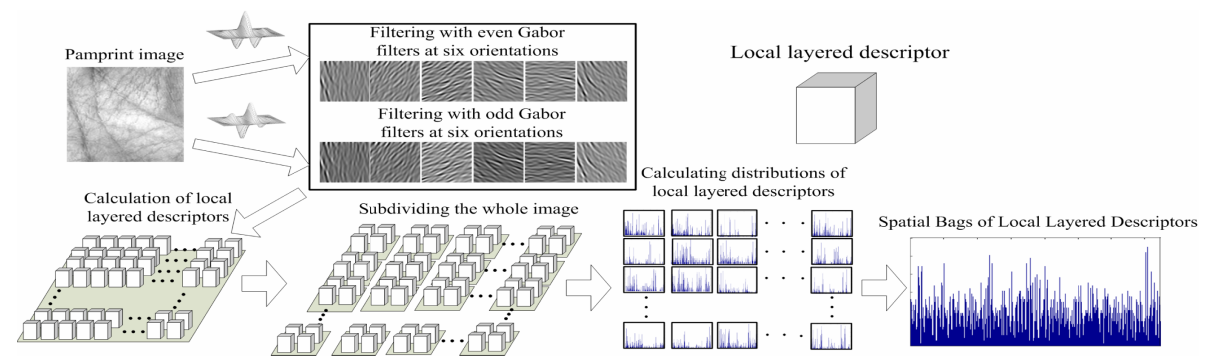

Fig. 2. Construction of SBLLD based palmprint representation

\section{Description of SBLLD Scheme}

\subsection{Local Layered Palmprint Descriptor}

In low resolution palmprint images, line segments and textures are observable and discriminative features [2]. Theoretically, quadrature Gabor filter provides accurate time-frequency location and good orientation selectivity to describe both of palmprint patterns within local image patches. A complex quadrature Gabor function is shown in Eq. 1

$$
G(x, y, \theta, u, \sigma)=\frac{1}{2 \pi \sigma^{2}} \exp \left(-\frac{x^{2}+y^{2}}{2 \sigma^{2}}\right)(\cos (2 \pi u \tilde{\theta})+i \sin (2 \pi u \tilde{\theta}))
$$

where $\tilde{\theta}=x \cos \theta+y \sin \theta$ and $i=\sqrt{-1} . u$ and $\sigma$ are the frequency of sinusoid wave and the standard deviation of Gaussian envelope. The real part of the function, namely even Gabor filter, optimally models directional line-like patterns such as wrinkles and principle lines in palmprints. Meanwhile, the imaginary part, namely odd Gabor filter, approximates the 1st-order directional derivatives of intensities. By constructing odd Gabor filter templates, we can obtain good estimation of image gradient, which is an efficient indicator of local image textures [8][9]. In previous work, Zhang and 
Kong et al [5][6] use Gabor filter sets to extract quantified phase features of palmprint images. Kong et al [6] combines multi-directional even Gabor filters and a "winnertake-all" scheme to describe direction of line segments in palmprints. Their results denote efficiency of quadrature Gabor filter in palmprint analysis. Motivated by properties of the filter, we construct a two-layered descriptor $T$ to depict both of discriminative palmprint patterns inside a local image patch, using quadrature Gabor filters at different orientation bands. Fig.3 illustrates structure of $T$.

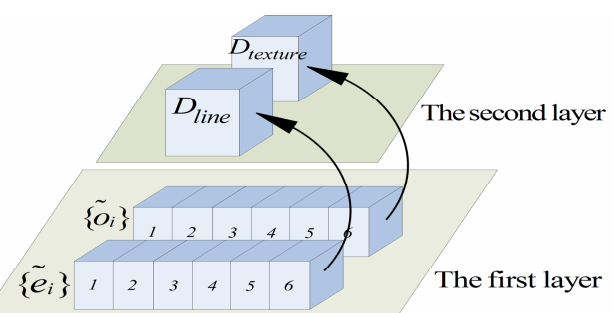

Fig. 3. The structure of two-layered descriptor

Before construction of the local descriptor, we adopt quadrature Gabor kernels with zero DC values at six orientations $\theta \in\{0, \pi / 6, \pi / 3, \pi / 2,2 \pi / 3,5 \pi / 6\}$. The parameter $u$ and $\sigma$ of them equal to 0.0916 and 5.6179. The size of each filter is $35 \times 35$. Empirical analysis testifies that this setting is suitable for palmprint recognition [5][6]. By performing convolution on a local patch with those Gabor kernels, totally 12 filtering responses $\left\{e_{i}, o_{i}(i=1,2 \ldots 6)\right\}$ are obtained. $e_{i}$ and $o_{i}$ are filtering responses of the even and odd Gabor filter respectively, as defined in Eq.2.

$$
\begin{aligned}
& e_{i}=P(x, y) * \operatorname{Re}\left\{G\left(x, y, \frac{(i-1) \pi}{6}, u, \sigma\right)\right\} \\
& o_{i}=P(x, y) * \operatorname{Im}\left\{G\left(x, y, \frac{(i-1) \pi}{6}, u, \sigma\right)\right\}
\end{aligned}
$$

where $\operatorname{Re}\{G\}$ and $\operatorname{Im}\{G\}$ represent even and odd Gabor filter. Elements of the first layer in the descriptor is then derived by applying ordinal encoding procedure [7] on original filtering responses, as shown in Eq.3.

$$
\begin{cases}\widetilde{e_{\mathrm{i}}} \text { or } \widetilde{o}_{\mathrm{i}}=1 & \text { if } e_{\mathrm{i}}>0 \text { or } o_{\mathrm{i}}>0 \\ \widetilde{e_{\mathrm{i}}} \text { or } \tilde{o_{\mathrm{i}}}=0 & \text { if } e_{\mathrm{i}}<0 \text { or } o_{\mathrm{i}}<0\end{cases}
$$

where $\tilde{e}_{\mathrm{i}}$ and $\widetilde{o_{\mathrm{i}}}$ are ordinal codes of corresponding filtering results. Ordinal encoding scheme [10] improves robustness of filter based appearance descriptions against changes of illumination settings and indicates intrinsic structures of lines and texture patterns. Thus, as we see in Fig.3, the first layer is defined as a 2nd-order tensor (matrix), with $\left\{\tilde{e_{\mathrm{i}}}\right\}$ and $\left\{\tilde{o_{\mathrm{i}}}\right\}(i=1,2 \ldots 6)$ as its row vectors. The former one is used as robust line description in the descriptor, while the latter one is utilized for depicting 
textures in palmprints. Compared with the first layer, the second one contains more abstract characteristics of appearances within local regions. Based on $\left\{e_{i}\right\}$ and $\left\{o_{i}\right\}$, we further calculate quantified orientation of prominent line patterns $D_{\text {line }}$ (ranging from 1 to 6), which equals to the index of response $e_{i}$ that achieves the minimum magnitude among $\left\{e_{i}\right\}(i=1,2 . .6)$ [6]. This orientation information plays an important role in accurate description of palm lines [6]. For describing texture patterns of palmprints, the approximated direction of image gradient $D_{\text {texture }}$ (ranging from 1 to 6 ) is estimated from $\left\{o_{i}\right\}(i=1,2 . .6)$ [8]. According to Eq.4, $D_{\text {texture }}$ is defined as the index of the angle band which has the strongest contrast response.

$$
\begin{aligned}
& D_{\text {line }}=\arg \min _{i} e_{i} \\
& D_{\text {texture }}=\arg \max _{i}\left|o_{i}\right|
\end{aligned}
$$

The second layer is then composed by a $2-\mathrm{D}$ vector $\{\mathrm{D}$ line, Dtexture $\}$, which corresponds to directional feature of both palmprint patterns. Through the layered scheme, we can achieve a comprehensive description of local regions in palmprint images. Firstly, in the top layer, we extract abstract description of discriminative palmprint patterns based on the effective directional information. Secondly, in the bottom layer, we make use of robust ordinal features to depict local appearances of line segments and textures in a more comprehensive manner. In our work, we sample a palmprint image densely. As a result, for a $\mathrm{h}^{\mathrm{W}_{\mathrm{w}}}$ image, totally $\mathrm{h} \times_{\mathrm{w}}$ local layered descriptors are derived.

\subsection{Formulation of Tree-Structured Codebook}

In our application, we adopt an efficient coding scheme to map layered descriptors to leaf nodes of a tree-structured codebook, which is consistent with formation of the descriptors. Based on this scheme, we can obtain visual codes fast using a look-up table, without prior clustering [10] performed in classic Bag-of-Feature model. The whole codebook contains three stages, as shown in Fig.4. On the root stage, the codebook $C$ is divided into two non-overlapped sub-codebooks $S_{\text {line }}$ and $S_{\text {texture }}$, which correspond to representations of line segments or texture patterns in palmprints. They are used in parallel during encoding visual patterns. In the following stage, each subcodebook is partitioned into six branches, indexed by integers from 1 to 6 , which correspond to values of $D_{\text {line }}$ or $D_{\text {texture }}$ in the local descriptors. Within a given branch, totally 63 leaf nodes transverse all possible combinations of six ordinal codes $\left\{\tilde{e_{i}}\right\}$ or $\left\{\tilde{o_{\mathrm{i}}}\right\}$. Given a fixed $D_{\text {line }}$ or $D_{\text {texture }}$, a descriptor is mapped to a leaf node by computing a label $L_{\text {leaf }}$ following Eq.5.

$$
L_{\text {leaf }}=\sum_{i=1}^{6} 2^{i-1} \tilde{e}_{i} \text { or } L_{\text {leaf }}=\sum_{i=1}^{6} 2^{i-1} \tilde{o}_{i}
$$

According to structure of the codebook, each local layered descriptor corresponds to two leaf nodes in the codebook, which belong to $S_{\text {line }}$ and $S_{\text {texture }}$ respectively. Moreover, directional information of lines and texture patterns has a dominant position in the palmprint description, which is consistent with verified conclusions in palmprint 
recognition [5][6]. With the same orientation label, quantified outputs of quadrature Gabor kernels represent finer differences of appearances within local patches, in order to provide helpful supplements for accurate palmprint representation.

As we can see in Fig.4, there are totally 768 leaf nodes in the codebook, which cover all possible visual patterns that one local layered descriptor can represent, without considering practical significance of each pattern in palmprint analysis. Actually, some patterns in the sub-codebooks $S_{\text {line }}$ are surplus. For example, assuming that $D_{\text {line }}$ equals to 1 , filtering respones $\tilde{e_{1}}$ is negative for sure. Thus, any pattern with the first element in $\left\{\tilde{e_{\mathrm{i}}}\right\}$ equaling to 1 is invalid for palmprint description. We remove such surplus patterns from the codebook. As a result, there are 582 nodes left to form a more reasonable and compact codebook for palmprint description.

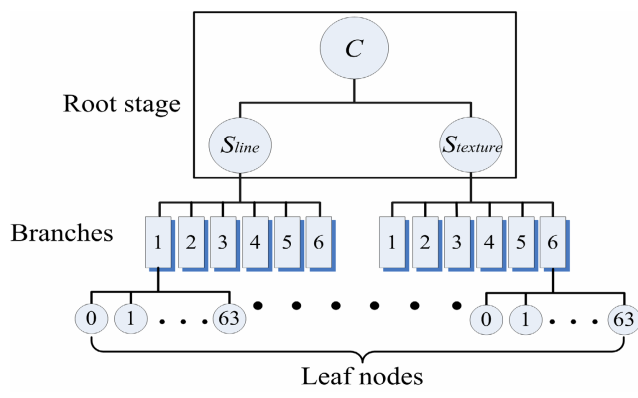

Fig. 4. The hierarchical structure of visual codebook

\subsection{Subdividing Scheme}

Although holistic image model is robust enough to local appearance deformation, it loses all spatial information of local features, making the image representation not suitable for accurate palmprint classification. In our method, we partition the whole image into sub-regions or spatial bags, and compute histograms of layered descriptor inside each sub-region. The proposed SBLLD is then constructed by aggregating histograms into a sequence according to their spatial order. Chi-square distance between SBLLD features is taken as the similarity measure. This subdividing scheme [12] has been practiced efficiently in computer vision, both for global and local image model. By dividing images into sub-blocks, we obtain distribution information of local descriptors at finer scale, which improves the ability of the statistical image representation to depict local details. Scale of partitioning is the key factor in the scheme. With increasing finer scale, the proposed representation becomes more sensitive to local appearance variations, which leads reduction of robustness to local appearance variations and higher dimensionality in the obtained histogram sequences. Therefore, to achieve an optimal trade-off, we should choose a proper size of individual sub-block. Experiments in Section 4 will give a detailed analysis on this issue. 


\section{Ranking Based Feature Selection}

A typical SBLLD usually has high dimensional histogram based representation. In theory, higher dimension can provide more discriminating power. However, it can also introduce non-informative attributes into the histogram vector of SBLLD, which are irrelevant with category information. In SBLLD, each dimension of the histogram corresponds to one visual pattern in a specific region of palmprints. Nevertheless, not all patterns provide efficient information for classification. For example, patterns appearing in different palmprint images with almost equal frequencies contain little discriminative power. Moreover, random image noise can also generate meaningless patterns. This is not a completely brand-new problem. Similarly in text categorization, large amount of vocabulary in corpus always leads to high dimensional histograms of term (word or phrase) occurrence [11]. To remove non-informative elements, all terms in texts are sorted and listed according to a term-goodness based criterion, such as information gain and document frequency [11]. Then a desired degree of terms at back of the list are eliminated from the vocabulary. The ranking based approach is suitable for high dimensional data due to its linear time complexity in terms of dimensionality. Simple as it is, it is efficient in selecting a well suited feature subset for classification [11]. Motivated by this idea, we perform feature selection based on a classification oriented criterion, rather than those general ones. Each dimension of the histogram vector is treated as one feature in SBLLD model. For each feature $i$, we define a stump classifier $C(i)$ based on normalized difference of corresponding dimension in two different SBLLD feature vectors $A$ and $B$, as denoted by Eq.6. In the expression, $T$ is a per-determined threshold. By performing an optimal threshold on outputs of $C(i)$, we can obtain the minimum classification error (MCE) of $C(i)$, which is used to evaluate predicting power of each single feature. With a set of labeled training data, the optimal threshold $t_{\text {optimal }}$ is obtained following Eq.7.

$$
\begin{gathered}
C(i)=\operatorname{sgn}\left(\frac{|A(i)-B(i)|}{A(i)+B(i)}-t\right) \\
C(i)= \begin{cases}1 \quad A \text { and } B \text { belong to the same class } \\
-1 \quad A \text { and } B \text { belong to different classes }\end{cases} \\
E=\frac{\text { False rejected items }+ \text { False accepted items }}{\text { Total amounts of matching }} \\
t_{\text {optimal }}=\arg \min _{t} E
\end{gathered}
$$

where $E$ is classification error corresponding to a specific threshold. All features are sorted in an ascending order according to MCE of each $C(i)$. The lower the minimum error is, the more relevant the specific feature is to palmprint classification. The optimal subset consists of top $K$ items in the ranking list. Lower $K$ leads to more compact representation. However, it may also exclude useful terms. In the following section, we choose a proper value of $K$ to achieve the best performance. 


\section{Experiments on PolyU Palmprint Database Version 2.0}

We compare performances of the proposed method on PolyU Palmprint Database Version 2.0 [13]. This database contains 7,752 384*286 palmprint images, which are captured from 386 palms. Each palm has two sessions of images, either of which contains more than 9 images. Average time interval between two sessions is two months, which introduce changes of light conditions, focus of the imaging device and deformation of skin textures due to varied hand postures between two occasions of image capturing [2], as shown in Fig.5. In our experiments, our proposed method is compared with four state-of-the-art component based algorithms [5][6][7] through one-to-one verification test.

After preprocessing, regions of interests, with the size of $128 \times 128$ are obtained. In this section, we implement SBLLD by dividing the whole ROI into $8 * 8,16 * 16$ and $32 * 32$ non-overlapped blocks, named as $S B L L D 8, S B L L D_{16}, S B L L D_{32}$ respectively, which cover descriptions from coarse scale to fine spatial scale. As we see in Table.1, although $S B L L D_{8}$ achieves the highest accuracy, improvement of performance is limited compared with $S B L L D_{16}$. Too fine spatial scale in division leads to increasing sensitivity to local appearance changes, which conversely deteriorates descriptive power of features. It also results in too high dimensional (148,992 bins) histogram vectors of $S B L L D_{8}$ requiring much computational cost. Thus, we choose $S B L L D_{16}$ to obtain a good trade-off. With the optimal division scheme, the SBLLD achieves satisfied performance, which is similar as the highest recognition accuracy of the state-ofthe-art methods. The histogram vector of $S B L L D 16$ has overall 37,248 bins, with each bin treated as an attribute in the representation. As described in Section 3, we further

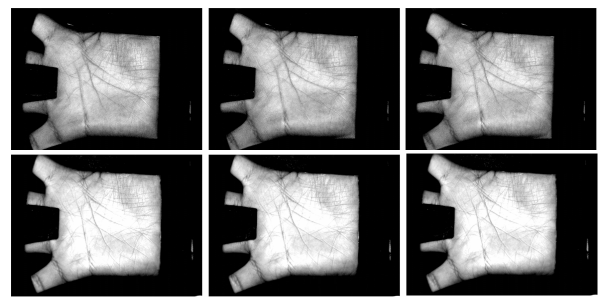

Fig. 5. Six examples of captured images in PolyU Palmprint Database Version 2.0

Table 1. Performances on the PolyU Database with different sub-dividing schemes

\begin{tabular}{|c|c|c|}
\hline & $d^{\prime}[14]$ & EER[14] \\
\hline$S B L L D_{8}$ & 4.16 & $0.038 \%$ \\
\hline$S B L L D_{16}$ & 4.22 & $0.049 \%$ \\
\hline$S B L L D_{32}$ & 3.99 & $0.581 \%$ \\
\hline Ordinal code [7] & 6.90 & $0.052 \%$ \\
\hline Competitive code [6] & 5.84 & $0.045 \%$ \\
\hline Fusion code [5] & 5.40 & $0.217 \%$ \\
\hline
\end{tabular}


Table 2. Verification performances with different choices of $\mathrm{K}$ on the testing set

\begin{tabular}{|c|c|c|}
\hline & $d^{\prime}[14]$ & EER[14] \\
\hline$K=5000$ & 4.78 & $0.048 \%$ \\
\hline$K=7000$ & 4.69 & $0.039 \%$ \\
\hline$K=10000$ & 4.57 & $0.026 \%$ \\
\hline$K=13000$ & 4.49 & $0.016 \%$ \\
\hline$K=15000$ & 4.43 & $0.017 \%$ \\
\hline$K=17000$ & $\mathbf{4 . 4 0}$ & $\mathbf{0 . 0 1 1 \%}$ \\
\hline$K=20000$ & 4.34 & $0.015 \%$ \\
\hline$K=23000$ & 4.30 & $0.012 \%$ \\
\hline$K=25000$ & 4.27 & $0.017 \%$ \\
\hline$K=27000$ & 4.25 & $0.020 \%$ \\
\hline$K=30000$ & 4.22 & $0.025 \%$ \\
\hline$K=33000$ & 4.21 & $0.028 \%$ \\
\hline$K=37248$ & 4.22 & $0.051 \%$ \\
\hline
\end{tabular}

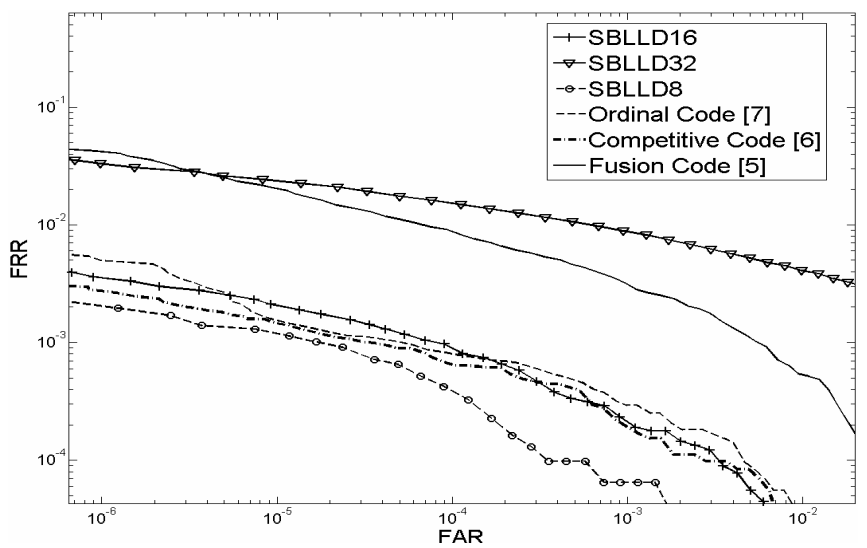

Fig. 6. ROC curves with different sub-dividing schemes

sort all attributes in descending order according to their predicting power measured on a training data set which is composed by four images randomly selected from each class in PolyU Database. Left 6,208 images form a testing set. The top $K$ attributes in the sorted list compose a subset $S$. Chi-square distance between tailored histogram vectors, which only reserve attributes in $S$, is defined as the similarity measure. We test the proposed tailored palmprint representation on the testing set. As we can see in Table.2, less $K$ leads to more strict selection. Aggressive choice of $K$, e.g. less than 5000 , even excludes informative features from the representation, which results in much performance deterioration of the proposed method. By increasing $K$ gradually from 1000 to 10000 , performance of our method is improved greatly. During further increasing from 10000 to 17000 , improvement of recognition accuracy becomes gentler, which confirms that leading portions of the ranking list contribute much more in classification than the posteriors. Conversely, $K$ larger than 20000 doesn't enhance 
classification ability stably or even worse. It verifies that attributes at the back of the list are usually irrelevant with classification task. They are generated by noninformative patterns in palmprint images. As a result, they provide no more stable clues for recognition or even decrease differences between inter-class samples. As we can see in Table.2, $K$ equaling to 17000 leads to the best performance on test data set, much higher than using all original attributes. By proper removal of irrelevant terms, the proposed SBLLD model can achieve efficient palmprint recognition, even more accurate than state-of-the-art methods.

\section{Conclusion}

In this paper, we have proposed a novel statistical palmprint representation, called Spatial Bags of Local Layered Descriptor (SBLLD). In this method, by integrating local distributions of local layered descriptors and their spatial order, we obtain accurate description of discriminative palmprint patterns, such as lines and textures on skin surfaces of palm regions, which consistently achieves accurate palmprint recognition. By performing the ranking based feature selection on the proposed statistical image representation, our proposed method obtains great improvement of performance with more compact histogram feature vectors, even better than most of state-ofthe art methods using component based texture analysis. However, the proposed model still needs additional storage and computation cost to construct and store high dimensional features. This problem has not been addressed well. In the future work, we will focus on this issue following the proposed framework.

\section{Acknowledgement}

This work is supported by research grants from the National Basic Research Program (Grant No.2004CB318110), the Natural Science Foundation of China (Grant No.60723005, 60736018, 60702024), NLPR 2008NLPRZY-2, the National Hi-Tech Research and Development Program of China (2006AA01Z193,2007AA01Z162).

\section{References}

[1] Jain, A.K., Bolle, R.M., Pankanti, S. (eds.): Biometrics: Personal Identification in Networked Society. Kluwer, Norwell (1999)

[2] Zhang, D., Kong, W.K., You, J., Wong, M.: Online Palmprint Identification. IEEE Transactions on Pattern Analysis and Machine Intelligence 25(9), 1041-1050 (2003)

[3] Wu, X.Q., Wang, K.Q., Zhang, D.: Palmprint Recognition using Directional Line Energy Feature. In: Proceedings of the 17th ICPR, vol. 4, pp. 475-478 (2004)

[4] You, J., Kong, W.K., Zhang, D., Cheung, K.: On Hierarchical Palmprint Coding with Multi-features for Personal Identification in Large Databases. IEEE Transactions on Circuit Systems for Video Technology 14(2), 234-243 (2004)

[5] Kong, W.K., Zhang, D.: Feature-Level Fusion for Effective Palmprint Authentication. In: Zhang, D., Jain, A.K. (eds.) ICBA 2004. LNCS, vol. 3072, pp. 761-767. Springer, Heidelberg (2004) 
[6] Kong, W.K., Zhang, D.: Competitive Coding Scheme for Palmprint Verification. In: Proceedings of the 17th ICPR, vol. 1, pp. 520-523 (2004)

[7] Sun, Z.N., Tan, T.N., Wang, Y.H., Li, S.Z.: Ordinal Palmprint Representation for Personal Identification. In: Proceedings of CVPR 2005, vol. 1, pp. 279-284 (2005)

[8] Han, Y.F., Sun, Z.N., Tan, T.N.: Palmprint Recognition Based on Directional Features and Graph Matching. In: Lee, S.-W., Li, S.Z. (eds.) ICB 2007. LNCS, vol. 4642, pp. 1164-1173. Springer, Heidelberg (2007)

[9] Chen, H.F., Belhumeur, P.N., Jacobs, D.W.: In Search of Illumination Invariants. In: Proceedings of CVPR 2000, pp. I:254-261 (2000)

[10] Leung, T., Malik, J.: Representing and recognizing the visual appearance of materials using three-dimensional textons. International Journal of Computer Vision 43, 29-44 (2001)

[11] Yang, Y., Pedersen, J.O.: A Comparative Study on Features Selection in Text Categorization. In: Proceedings of the 14th ICML, pp. 412-420 (1997)

[12] Lazebnik, S., Schmid, C., Ponce, J.: Beyond Bags of Features: Spatial Pyramid Matching for Recognizing Natural Scene Categories. In: Proceedings of CVPR 2006, vol. 2, pp. 2169-2178 (2006)

[13] PolyU Palmprint Database, http: / /www . comp. polyu. edu.hk/ biometrics /

[14] Daugman, J., Williams, G.: A Proposed Standard for Biometric Decidability. In: Proceedings of CardTech/ SecureTech Conference, Atlanta, GA, pp. 223-234 (1996) 